\title{
Dynamic Schema Hierarchies for an Autonomous Robot
}

\author{
José M. Cañas ${ }^{1}$ and Vicente Matellán ${ }^{1}$ \\ Universidad Rey Juan Carlos, 28933 Móstoles (Spain) \\ $\{$ jmplaza, vmo\}@gsyc.escet.urjc.es
}

\begin{abstract}
This paper proposes a behavior based architecture for robot control which uses dynamic hierarchies of small schemas to generate autonomous behavior. Each schema is a flow of execution with a target, can be turned on and off, and has several parameters which tune its behavior. Low level schemas are woken up and modulated by upper level schemas, forming a hierarchy for a given behavior. At any time there are several awake schemas per level, running concurrently, but only one of them is activated by environment perception. When none or more than one schema wants to be activated then upper level schema is called for arbitration. This paper also describes an implementation of the architecture and its use on a real robot.
\end{abstract}

\section{Introduction}

It is not fiction science to see a mobile robot guiding through a museum ${ }^{1}$, navigating in an office environment, serving as a pet $^{2}$, or even playing soccer ${ }^{3}$. Today we've got best sensors and actuators ever. But how are they combined to generate behaviors?

The hardware improvements have made clear the importance of a good control architecure, making it a critical factor to obtain the goal of autonomous behavior. Robot control architecture can be defined as the organization of robot sensory, actuation and computing capabilities in order to generate a wide set of intelligent behaviors in certain environment. Architecture answers to two main questions: what to do next? (action selection) and what is interesting in the environment? (attention). Advances in architecture will lead to even more complex behaviors and increasing reliable autonomy.

In next section main ideas and proposals in robot control architectures will be reviewed. Section 3 presents Dynamic Schema Hierarchies including its action selection mechanism, its reconfiguration habilities and how perception is organized in this proposal. The software architecture developed to implement DSH is commented on section 4 . Some conclusions and future lines end the paper.

\footnotetext{
${ }^{1}$ Minerva: http://www.cs.cmu.edu/ minerva

2 Aibo:http://www.aibo.com

${ }^{3}$ Robocup: http://www.robocup.org/
} 


\section{Robot Control Architectures}

\subsection{Deliberative Architectures}

Symbolic AI has influenced on mobile robotics from its beginnings, resulting in deliberative approach. This makes emphasis on world modelling and planning as deliberation for robot action. In mid eighties this was the main paradigm for behavior generation. The control architecture was seen as an infinite information loop: Sense-Model-Plan-Act (SMPA). In modelling step sensor data are fused into a central world representation, which stores all data about environment, maybe in a symbolic form. Most robot intelligence lie in the planning step, where a planner searched in the state space and found a sequence operators to reach some target state from the current one. Act were seen as a mere plan execution.

There was a single execution flow and a functional decomposition of the problem, where the modules called functions from other modules (vision module, path planning module). The functional decomposition prevented a incremental development: all the modules had to be implemented to start having some behavior.

\subsection{Behavior Based Architectures}

Rooted in connectionist theories in mid eighties new approaches which exhibited impressive demos on real robots were proposed. The common factor of such works was the distribution of control in several basic behavior units, called levels of competence, schemas, agents, etc. .

Each behavior unit is a fast loop from sensor to action, with its own partial target. There is no central representation, each behavior processes its own sensory information. Additionally, there are no explicit symbols about the environment. The emphasis were put in real world robots (embodiment) and in interaction with the environment (situated).

Distribution of control poses the additional problem of behavior coordination. Each behavior has its own goal, but usually enters in contradiction with another one. How is the final actuation calculated (action selection)?. There are two major paradigms: arbitration and command fusion. Arbitration stablishes a competition for control among all the behaviors and only the winning one determines the final actuation. Priorities, activation networks from Pattie Maes [12] and state based arbitration [3] fall in this category. Command fusion merge all the relevant outputs in a global one that take into account all behavior preferences. Relevant approaches in command fusion include superposition[2], fuzzy blending [14] and voting [13]. Coordination is always a difficult issue.

We will present here in more detail on two foundational works leaving apart extensions and refinements. They contain main relevant ideas of the approach.

Brook's subsumption In 1986 Rodney Brooks proposed a layered decomposition of behavior in competence levels [6]. Since then many robots have been developed using this paradigm (Herbert, Toto), showing a great proficiency in 
low level tasks such as, trash cans collecting, etc.. A competence level is an informal specification of a desired class of behaviors for a robot. Each level is implemented by a net of Finite State Machines (FSM), which have low bandwidth communication channels to exchange signals and small variables.

Low levels provide basic behaviors, i.e. avoid obstacles, wander, etc.. More refined behaviors are generated building additional levels over the existing ones, which don't know anything about the new ones. All levels run concurrently, and upper levels can supress lower level outputs and replace their inputs. This is called subsumption and gives name to the architecture. This action selection mechanism uses fixed priorities hardwired in the FSM net.

Arkin' schemas Following Arbib ideas [1], Ronald Arkin proposed a decomposition of behavior in schemas [2]. His architecture, named AuRA, contained two types of units: motor schemas and perceptive ones. "Each motor schema has an embedded perceptual schema to provide the necessary sensor information" [2].

For instance, the output of a navigation motor schema is a vector with the desired velocity and orientation to advance. The navigation behavior was obtained by the combination of avoid-moving-obstacles, avoid-static-obstacles, stay-onpath and move-to-goal schemas. Each schema can be implemented as a potential field, delivering a force vector for each location in the environment. The commanded movement is the superposition of all fields [2].

Extensions to Arkin's approach include the sequencing of several complex behaviors, each one composed of concurrent activation of several schemas. A Finite State Acceptor is used for arbitration, where each state means the activation of certain schemas and triggering events are defined to jump among states [3].

\subsection{Hybrid approaches}

The trend in last years is an evolution to hybrid architectures that combine the strenghs of both paradigms. For instance planning capabilities and fast reactivity, because they both are important for complex tasks on real reliable robots.

A succesful approach is the layered 3T-architecture [5], based on Firby's RAP [9]. The control is distributed in a fixed hierarchy of three levels of abstraction that run concurrently and asynchronously. Upper layer includes deliberation over symbolic representations and makes plans composed of tasks. The intermediate level, called sequencer, receives such tasks and has a library of task recipes describing how to achieve them. It activates and deactivates sets of skills to accomplish the tasks. Each skill is a continuous routine that achieve or maintain certain goal in a given context (it is situated). All skills lie in the bottom level, the reactive layer.

\section{Dynamic Schema Hierarchies}

We propose an approach named Dynamic Schema Hierarchies (DSH) that is strongly rooted in Arbib [1] and Arkin ideas [2]. The basic unit of behavior is called schema. Control is distributed among a hierarchy of schemas. 
An schema is a flow of execution with a target. It can be turned on and off, and accepts several input parameters which tune its own behavior. There are perceptual schemas and motor schemas. Perceptual ones produces pieces of information that can be read by other schemas. These data usually are sensor observations or relevant stimuli in current environment, and they are the input for motor schemas. Motor schemas access to such data and generate their outputs, which are the activation signal for other low level schemas (perceptual or motor) and their modulation parameters.

All schemas are iterative processes, they perform their mission in iterations which are executed periodically. Actually, the period of such iterations is a main modulation parameter of the schema itself. Digital controllers are an example of such paradigm, they deliver a corrective action each control cycle. Schemas are also suspendable, they can be deactivated at the end on one iteration and they will not produce any output until they are resumed again.

A perceptual schema can be in only two states: SLEPT or ACTIVE. When ACTIVE the schema is updating the stimuli variables it is in charge of. When SLEPT the variables themselves exist, but they are outdated. The change from SLEPT to ACTIVE or viceversa is determined by upper level schemas.

For motor schemas things are a little bit more tricky, they can be in four states: SLEPT, CHECKING, READY and ACTIVE. A motor schema usually has preconditions, which must be satisfied in order to be ACTIVE. CHECKING means the schema is awake and actively checking its preconditions, but they don't match to current situation. When they do, the schema passes to READY and tries to win action selection competition against other READY motor schemas in the same level and so become ACTIVE. Only ACTIVE schemas deliver activation signals and modulation parameters to lower level schemas.

Schemas can be implemented with many different techniques: simple conditions from sensor data, fuzzy controllers, planners, finite state machines, etc. . The only requirement is to be iterative and suspendable. In the case of a planner, the plan is enforced to be considered a resource, an internalized plan [13] instead of a symbolic one. This is because the schema has to deliver an action proposal each iteration.

\subsection{Hierarchy}

Schemas are organized in hierarchies. This hierarchies are dinamically built. For instance, an ACTIVE motor schema needs some information to accomplish its target. In order to collect, search, build and update such information it activates relevant perceptual schemas (square boxes in figure 1). It may also awake a set of low level motor schemas (circles) that can be useful for its purpose because implement right reactions to stimuli in the environment. It modulates them to behave according to its own target and put them in CHECKING state. Not only the one convenient for current situation but also all the lower motor schemas which deal with plausible situations. This way low level schemas are recursively woken up and modulated by upper level schemas, forming a unique hierarchy for a given global behavior. 
At any time there are several CHECKING motor schemas running concurrently per level, displayed as solid circles in figure 1 (i.e. schemas 5, 6 and 7). Only one of them per level is activated by environment perception or by explicit parent arbitration, as we will see on 3.2. The ACTIVE schemas are shown as filled circles in figure 1 (1,6 and 15). For instance motor schema 6 in figure 1 is the winner of control competition at the level and is the only one that awakes lower level parameters. It awakes perceptual schemas 11, 16 and motor schemas 14, 15, and sets their modulation parameters.

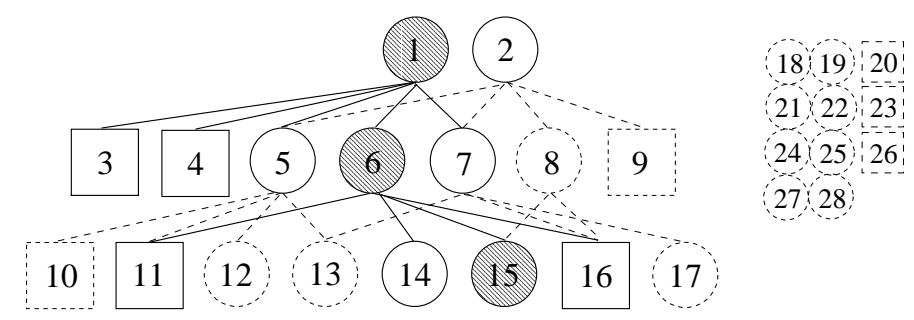

Fig. 1. Schema hierarchy and pool of SLEPT schemas

Schemas unused for current task rest in a pool of schemas, suspended in SLEPT state, but ready for activation at any time. They appear as dashed squares and circles in figure 1 (schemas $8,9,10,12,13,17,18$, etc.). Actually schemas 10,12 and 13 are one step away from activation, they will be awaken if schema 5 passed to ACTIVE in its level.

Sequence of behaviors can be implemented with DSH using a motor schema coded as a finite state machine. Each state corresponds to one step in the sequence, and makes a different set of lower schemas to be awaken. It also activates the perceptual schemas needed to detect triggering events that change its internal state.

\subsection{DSH Action Selection}

At any time the ACTIVE perceptual schemas draw a perceptual subspace that corresponds to all plausible values of relevant stimuli for each level (displayed as white areas in figure 2), that is, where is focused the attention. It is a subset of all possible stimuli, because it doesn't include the stimuli produced by SLEPT perceptual schemas (shadowed area in figure 2).

This subspace is partitioned into activation regions, which are defined as the areas where the preconditions of a motor schema are satisfied. Usually an ACTIVE motor schema awakes several perceptual schemas on lower level and several motor schemas, which are taken from the pool and passed to CHECKING state. These child motor schemas are modulated to be roughly disjointed on the perceptual subspace, that is, to have non overlapping activation regions. 
A given situation corresponds to one point in such subspace, and may lie in the activation region of one motor schema or another. Only the corresponding motor schema will be activated, so situation activates only one schema per level among CHECKING ones. This is a coarse grained arbitration based on activation regions.

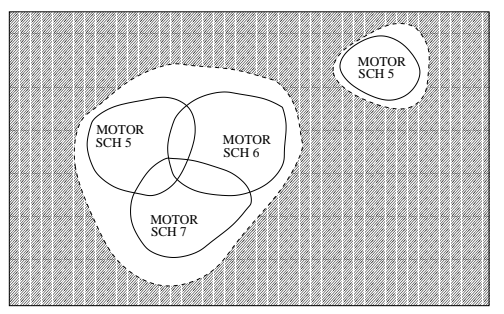

Fig. 2. Perceptual space and activation regions

Despite such coarse grained arbitration may appear situations where more than one motor schema for that level satisfy their preconditions (activation regions overlap in figure 2). Or even situations where none of them are READY for activation, that is, not covered by any activation region. Child schemas detect such control failures checking their brothers' state, and then parent is called for fine grained arbitration. Parent can change children parameters or just select one of them as the winner of control competition is its level. This is similar to context dependent blending [14], the parent schema knows the context for that arbitration.

The action selection mechanism in DSH has a distributed nature. There is no central arbiter as in DAMN [10], just parent and child schemas. There is a competition per level, but there are no more levels than required by task. It also occurs once per any CHECKING motor schema iteration, because all of them check their own preconditions and the state of their brothers if needed. This allows a fast reconfiguration if situation changed.

It is a commitment between purposiveness top-down and reactive motivations. Only schemas awaken from upper level are allowed to gain control, but finally perceived situation chooses one and only one winner among them per level. The winner has double motivation, task-oriented and situation-oriented. Schemas without any of them don't add enough motivation for its activation and remain silent, CHECKING or SLEPT. Activation flows top-down in the hierarchy, similar to architecture proposed by Tinbergen and Lorenz [11] for instinctive behavior in animals. Addition of motivation and lateral inhibition among same level nodes also appear in such ethological architectures. 


\subsection{Perception}

In DSH motor schemas make their decisions over information produced by perceptual schemas. Perception is distributed in perceptual schemas, each one produces several pieces of such information. They exist because at least one motor schema eventually needs the information they produce. It can be sensor data that the schema collects, or more complex stimuli about environment or the robot itself that the schema builds, for instance a map, a door, etc.. All the events or stimuli that we want to take account of in the behavior require a computational effort to detect and perceive them. DSH includes them in the architecture as perceptual schemas, each one searches for and describes its stimulus when present, updating internal variables.

Due to hierarchical activation in DSH, perception is situated, context dependent. Perceptual schemas can be activated at will, the ACTIVE ones focus only on stimuli which are relevant to current situation or global behavior (attention). This filters out huge amounts of useless data from the sensors, i.e. shadowed area in figure 2 . It makes the system more efficient because no computational resource is devoted to stimuli not interesting in current context. Its corresponding perceptual schema is SLEPT or doesn't exist all.

There can be symbolic stimuli if they are convenient for the behavior at hand. These abstract stimuli must be grounded, with clear building and updating algorithms from sensor data or others lower level stimuli. Actually different levels in perceptual schemas allow for abstraction and dependent stimuli: perceptual schemas can have as input the output of other perceptual schemas.

\subsection{Reconfiguration}

For a given task certain hierarchy generates the right robot behavior. The net of schemas builds relevant stimuli from sensor data and reacts accordingly to environment state. If the situation changes slightly, currently ACTIVE schemas can deal with it and maybe generate a slightly different motor commands. If the situation changes a little bit more maybe one ACTIVE schema is not appropiate anymore and the environment itself activates another motor schema, that was ready to react to such change, in CHECKING.

In the case of bigger changes they cause a control conflict at certain level, the parent is called for arbitration and may decide to sleep useless schemas, wake up another relevant ones, change its children modulation or propagate the conflit upwards forcing a hierarchy reconfiguration from upper level. This reconfiguration may add new levels or reduce the number of them. This can be seen as a dynamic controller, composed of several controllers running in parallel and triggering events that change completely the controllers net. Each event requires a corresponding perceptual schema to detect it. This is similar to discrete state arbitration from [3], but accounts for hierarchy of schemas not only for a single level set of them.

The levels are not static but task dependent as in TCA task trees [15]. These changes must be designed to work properly. Actually schemas are placed in one 
level or another depending on the task at hand but schemas don't belong to any level in particular. They can be located in a different level, probably with other parameters, for another global behavior. This way the schemas can be reused in different levels depending on the desired final behavior.

\section{Implementation issues}

We have used a small indoor robot, composed of a pioneer platform and a offthe-shelf laptop under Linux OS, figure 3 . The robot is endowed with a 16 sonar belt, bumpers and two wheel encoders for position estimation. We have added a cheap webcam connected through USB to the computer. Two DC motors allow robot movements.
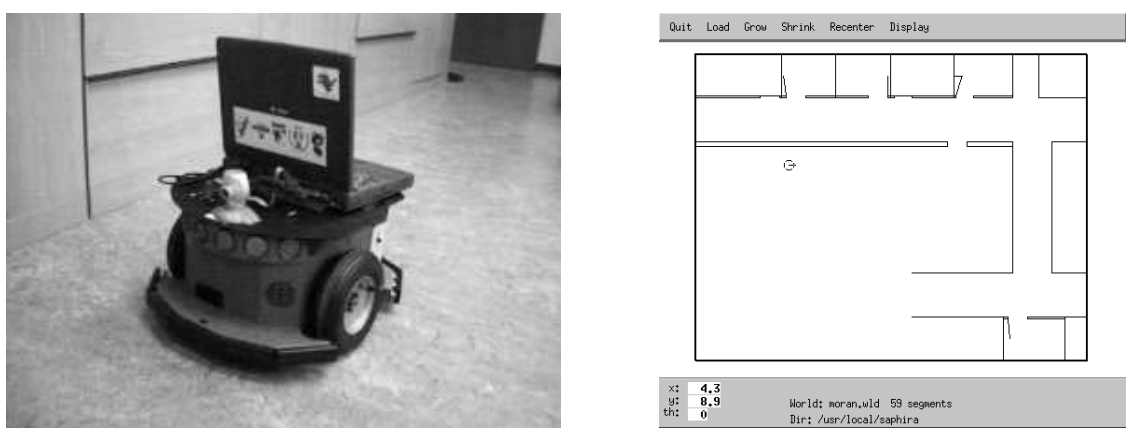

Fig. 3. Supercoco, our robot and saphira simulator

A software architecture has been developed to test the cognitive architecture proposed. All our code is written in ANSI-C. We have developed two socket servers which put camera images, motor commands, raw sonar, bumper and encoder data available for client programs through message protocol. These servers can connect both to robot simulator (saphira environment) or real plattform, so the same control program can run seemlessly on real robot and on the simulator (without vision). This is very convenient for debugging, crashes are painless in simulation!. Additionaly, the laptop is wireless connected, so the control program for real robot can run onboard or from any other computer in the net.

The DSH control program is a single client process, but with many kernel threads inside. Each schema is implemented as a thread (we use standard Posix threads on Linux), which periodically executes an iteration function. Communication between different threads is done through regular variables, because all the threads share virtual memory space. Using mutex motor schemas safely read variables updated by perceptual schemas, and low level schemas safely read their own parameters set by a higher level schema.

To implement SLEPT state we have used pthread condition variables. Each schema has an associated condition variable, so it can sleep on it when 
needed consuming no CPU cycles. Any schema may ask another one to be halted writing on a shared variable. The next time recipient execute its iteration it will be suspended on its condition variable. The schema can be resumed when another thread signal on its condition variable. Typically the parent schema sets the child parameters and then wakes it up signalling on its condition variable.

Arbitration functions are implemented as callback functions.

\subsection{Example}

Gotopoint behavior.

\section{Conclusions}

Deliberative approach has shown poor performance on real robots, specially in dynamic environments. Sensor data must pass through the complete series of SMPA stages before resulting in any action. This places an unconvenient delay in the loop between sensing and action. For instance the robot couldn't plan any action until more or less a complete model of the environment were available to reason about. In addition, modelling is a difficult task in real robots. It has to capture information from real sensors, usually uncertain, incomplete and noisy.

Despite simple behaviors on real robots as obstacle avoidance were built quite fast and proficiently, the approach doesn't scale for more complex behaviors and abstract stimuli.

General enough.

Problem of modifying a low level schema used by several high level schemas: do we need reprogramming such high level schemas? Old, and new low level schemas can coexist without any problem. High level schemas choose which one to activate, there is no need for reprogramming high level schemas.

This approach provides more flexibility than subsumption in one schema using low level ones and allows the incremental development in robot design. This approach provides more flexibility than subsumption and allows incremental development in robot design.

The architecture is extensible. Adding a new schema is quite easy, it requires to code a new-schema.h and a new-schema.c, declaring and defining the iteration function for that schema, an arbitration funtion (if needed) and an startup function.

More complex behaviors.

\section{References}

1. Arbib, M.A., Liaw, J.S.: Sensorimotor transformations in the worlds of frogs and robots. Artificial Intelligence, 72 (1995) 53-79

2. Arkin, R.C.: Motor Schema-Based Mobile Robot Navigation. The International Journal of Robotics Research, 8(4) (1989) 92-112 
3. Arkin, R.C., Balch, T.: AuRA: Principles and Practice in Review. Journal of Experimental and Theoretical Artificial Intelligence, 9(2-3) (1997) 175-188

4. Ali, K.S., Arkin, R.C.: Implementing Schema-theoretic Models of Animal Behavior in Robotic Systems. Proceedings of the 5th International Workshop on Advanced Motion Control, AMC'98. IEEE, Coimbra (Portugal) (1998) 246-253

5. Bonasso, R.P., Firby, R.J., Gat, E., Kortenkamp, D., Miller, D.P., Slack, M.G.: Experiences with an Architecture for Intelligent, Reactive Agents. Journal of Experimental and Theoretical Artificial Intelligence, 9(2) (1997) 237-256

6. Brooks, R.A.: A Robust Layered Control System for a Mobile Robot. IEEE Journal of Robotics and Automation, 2(1) (1986) 14-23

7. Corbacho, F.J., Arbib, M.A.: Learning to Detour. Adaptive Behavior, 5(4) (1995) 419-468

8. Firby, R.J.: Building Symbolic Primitives with Continuous Control Routines. Proceedings of the 1st International Conference on AI Planning Systems AIPS'92. (1992) 62-69

9. Firby, R.J.: Task Networks for Controlling Continuous Processes. Proceedings of the 2nd International Conference on AI Planning Systems AIPS'94. AAAI (1994) $49-54$

10. Langer, D., Rosenblatt, J.K., Hebert, M.: A Behavior-Based System for Off-Road Navigation. IEEE Journal of Robotics and Automation, 10(6) (1994) 776-782

11. Lorenz, K.: Foundations of Ethology. Springer Verlag (1981)

12. Maes, P.: How to Do the Right Thing. Connection Science Journal (Special Issue on Hybrid Systems), 1(3) (1989) 291-323

13. Payton, D.W., Rosenblatt, J.K., Keirsey, D.M.: Plan Guided Reaction. IEEE Transactions on Systems Man and Cybernetics, 20(6) (1990) 1370-1382

14. Saffiotti, A.: The uses of fuzzy logic in autonomous robot navigation. Soft Computing, 1 (1997) 180-197

15. Simmons, R.G.: Structured Control for Autonomous Robots. IEEE Transactions on Robotics and Automation, 10(1) (1994) 34-43

16. Tyrrell, T.: The Use of Hierarchies for Action Selection. Journal of Adaptive Behavior, 1(4) (1993) 387-420 